\title{
Outcome of heroin dependent adolescents presenting for opiate substitution treatment
}

\author{
Authors:- \\ Bobby P Smyth* MRCPsych, Department of Public Health \& Primary Care, Trinity College \\ Dublin, Ireland \\ John Fagan MRCPsych, Drug Treatment Centre Board, Dublin 2, Ireland. \\ Kathy Kernan MRCPsych, Drug Treatment Centre Board, Dublin 2, Ireland. \\ * Corresponding Author. \\ Bobby Smyth, Adolescent Addiction Service, Bridge House, Cherry Orchard Hospital, Dublin \\ 10 \\ Email: smythbo@tcd.ie \\ Phone: 0035316206409 \\ Fax: 0035316250752
}




\begin{abstract}
As the outcome of methadone and buprenorphine substitution treatment in adolescents is unclear, we completed a retrospective cohort study of 100 consecutive heroin dependent adolescents who sought these treatments over an 8 year recruitment period. Participants' average age was 16.6 years and 54 were female. Half of the patient group remained in treatment for over one year. Among those still in treatment at 12 months, 39\% demonstrated abstinence from heroin. The final route of departure from the treatment program was via planned detox for $22 \%$, drop out for $32 \%$ and imprisonment for $8 \%$. The remaining $39 \%$ were transferred elsewhere for ongoing opiate substitution treatment after a median period of 23 months treatment. Males were more likely to exit via imprisonment $(p<0.05)$, but other outcomes were not predicted by gender. There were no deaths during treatment among these 100 patients who had a cumulative period of 129 person years at risk. Our findings suggest that this treatment delivers reductions in heroin use and that one fifth of patients will exit treatment following detox completion within a one to two year time frame.
\end{abstract}




\section{Introduction}

Substitution treatment with agonist medications, such as methadone and buprenorphine, represents the treatment of choice for heroin dependent patients (NICE, 2007). The evaluations of methadone maintenance treatment (MMT) indicate that it reduces mortality, HIV related injecting risk behaviour, illicit heroin use and criminal activity (Marsch 1998; Marsden et al, 2009). Nevertheless, it is still surrounded by substantial political controversy and there is much legislation in many jurisdictions imposing limits on who can and cannot receive opiate substitution treatment (Fudala \& Woody, 2004).

Internationally, adolescent presentation to opioid treatment facilities is uncommon. In USA, $1 \%$ of treatment episodes in adolescents in 2007 related to heroin abuse (SAMSHA, 2009). In Britain, 3\% of treatment episodes in this age range in 2008-09 were precipitated by opiate abuse (NTA, 2009). Ireland witnessed a dramatic increase in heroin abuse during the mid1990s in Dublin, with adolescents accounting for $13 \%$ of all heroin related treatment attendances during that decade (Smyth \& O'Brien, 2004). As in Britain, the prevalence of heroin dependence in teenagers has diminished in recent years, with adolescents accounting for just $1 \%$ of heroin related addiction treatment episodes in 2007 (Carew et al, 2009). Opioid dependent patients in USA frequently present with abuse of prescription opioids (Motamed et al, 2008). This is uncommon in Ireland (Carew et al, 2009).

There is an absence of comprehensive international guidelines on use of opiate substitution treatment in adolescents. Guidelines from USA and Canada indicate that opiate substitution treatment should not be offered to adolescents unless they have previously failed a detoxification based treatment intervention (College of Physicians \& Surgeons of Ontario, 2005; Center for Substance Abuse Treatment; 2005). SAMSHA (2009) have suggested that opiate agonist treatments are possibly underutilised in this age group in the USA, with just 3\% 
of adolescent heroin admissions having such treatment as part of their care plan in 2007. In Britain, the Department of Health (2009) recently produced consensus guidelines on pharmacological treatments in addiction for adolescents, acknowledging a role for opiate agonist medications in this age range.

Although thoroughly evaluated in adults there has been surprisingly little research conducted on the outcomes of adolescents who receive this treatment (Fudala \& Woody, 2004; Hopfer et al 2002; Kaminer \& Marsch, 2011). There have been no large scale studies of methadone maintenance in adolescents since the 1970s. Millman, Khuri \& Nyswander (1978) reported outcome from their MMT program for 153 heroin dependent adolescents in New York in the 1970s. They found that $29 \%$ exited via planned detoxification, $21 \%$ either dropped out or faced a disciplinary discharge and $44 \%$ remained on MMT or were transferred elsewhere for ongoing treatment. Three percent died and 3\% went to prison. The DARP study was also conducted in USA and Sells \& Simpson (1979) reported outcome in a large subset of young people under the age of twenty years. They found reductions in heroin use in all treatment modalities and that MMT demonstrated better treatment retention than other treatment options. However, those who were retained in therapeutic community treatments did better than those retained in MMT in terms of occupational outcomes. While these studies provide some evidence of the effectiveness of methadone treatment in this age range, the findings may not generalise to modern cohorts of heroin dependent adolescents. In the intervening decades, awareness of HIV risks has occurred, misuse of prescription opiates has increased and alternative agonist treatments such as buprenorphine have become more widely used. These and other factors may have altered the patient profile, treatment provision and recovery context substantially in the past thirty-five years.

Minozzi, Amato \& Davoli (2009) completed a recent Cochrane review of opiate substitution treatment in adolescents but identified just two clinical trials in total (Woody et al, 2008; 
Lehman, 1973). The Lehman study compared LAAM to methadone but involved treatment of just four months and was judged to be "of very low quality", so no conclusions could be reached. While the Woody et al (2008) study was well designed and it examined buprenorphine treatment, it compared a two-week detox treatment against an extended 9 week treatment which then also ended in a detox. The treatment participants were generally young adults, with just $17 \%$ being under 18 years old. They reported reductions in opioid use and better treatment retention during the very brief maintenance phase of treatment, but both groups tended to quickly relapse following cessation of buprenorphine treatment. Therefore, this Cochrane review reveals very little about the outcome of sustained maintenance treatment in adolescents under the age of 18 years.

Since the 1970s, there have been a small number of relevant descriptive studies. Crome et al (2000) reported on the outcome of the first 48 patients with severe heroin dependence who were prescribed methadone at a youth treatment service in England. It was reported that $80 \%$ were retained in treatment and that $37 \%$ had a "good" outcome but the criteria utilised to determine outcome were subjective and somewhat unclear. A more recent Australian study highlighted the challenges of retaining this patient group in treatment (Bell \& Mutch, 2006). From the cohort of 61 teenagers, two-thirds dropped out of their index treatment episode. Among those who left treatment, following either detoxification or drop out, $62 \%$ subsequently re-accessed treatment.

The absence of recent relevant outcome research for this patient group means that there has been very little to guide clinicians, treatment commissioners, patients or parents about what to expect following commencement of an adolescent on opiate substitution treatment. We sought to address this gap in the scientific literature by providing a descriptive analysis of the experience of heroin dependent adolescents entering a substitution treatment program over an 
8-year period.. We also sought to examine incidence of hepatitis C, HIV and mortality among adolescents who commenced this treatment.

\section{Method}

\subsection{Setting}

In 2000, due to the large number of opiate dependent adolescents presenting to treatment services in Dublin, and the growing recognition that their treatment needs differed to that of the adult population, the Young Persons Program (YPP) was developed at the Drug Treatment Centre Board (DTCB). It provided a multi-disciplinary adolescent treatment service, working in a manner which was broadly consistent with the recent British guidelines (Department of Health, 2009). The team was led by a consultant child \& adolescent psychiatrist and had input from nursing, clinical psychology, social work, project workers, counsellors and family therapy. The staff to patient ratio was over double that which existed in the adult MMT programs in DTCB. The YPP was based in central Dublin and provided treatment to opiate dependent teenagers from all of Dublin, and the surrounding counties. Patients travelled up to 50 miles to attend the service.

Methadone prescribers are specifically regulated by legislation in Ireland (Butler, 2002). General Practitioners (GPs), or office based practitioners, are not permitted to initiate any patient onto methadone unless they have undergone substantial training, and all patients must be registered with the Central Treatment List. National guidelines on the treatment of addiction in adolescents emphasise the need for multidisciplinary input into treatment (Dept of Health \& Children, 2005). Consequently, GPs or office based practitioners, rarely prescribe methadone to heroin dependent adolescents. During the period of this study, only doctors working at the DTCB were permitted to prescribe buprenorphine in Ireland. In 2011, access to buprenorphine remains restricted to a small number of specialist addiction clinics in Dublin. 
Patients were expected to be aged 17 years or younger when they commenced assessment on the YPP, although 18 year olds were occasionally accepted. There was an expectation that patients progress from the service prior to their twentieth birthday. If there was a need for ongoing opiate substitution treatment, they were facilitated with a planned transfer to an adult addiction service.

\subsection{Assessment}

There was an open referral system to the YPP, with family and social workers being the most common sources of referral. There was no waiting list. There were no exclusion criteria for opiate dependent patients, although patients who were deemed to need acute inpatient treatment of a co-occurring medical or psychiatric disorder had their assessment postponed until this disorder was stabilised. Treatment was provided free of charge. Assessment occurred over a seven to ten day period, during which patients typically attended on three occasions to meet different members of the multi-disciplinary team. In addition to being assessed for heroin dependence using ICD-10 criteria, patients also provided three supervised urine samples as confirmatory evidence of regular heroin use. Assessment also sought to identify co-existing psychological, developmental, social and physical needs.

People aged 16 years and above may consent to medical treatment on their own behalf in Ireland (Dept of Health \& Children, 2005). However, we also sought parental agreement to treatment in these cases. Parental consent was required for patients under the age of 16 years. The main pillars of treatment involved opiate substitution medication (methadone or buprenorphine), counselling (CBT, motivational interviewing or humanistic person centred therapy) and family therapy in some cases. While patients were expected to attend counselling sessions on a weekly basis, failure to do so did not result in termination of treatment. In practice, it was our experience that about $50 \%$ of patients availed of individual counselling 
sessions per week. There were group activities during afternoons, using art therapy and delivery of life-skills. Attendance at groups was encouraged but not compulsory. Participation rates at groups were typically 40 to $60 \%$.

\subsection{Approach to Opiate Substitution Treatment}

Induction onto methadone typically commenced with a dose of $20 \mathrm{mgs}$ on day one. Dose was then increased by $10 \mathrm{mgs}$ every two to four days, titrated against withdrawal symptoms, cravings, ongoing heroin use, while also monitoring for evidence of sedation. Stabilisation doses were generally in the region between 40 and 70mgs. While this was lower than stabilisation doses typically recommended for adults, patients at the lower end of the dose spectrum had a personal preference for a relatively low dose and achieved stabilisation (i.e. absence of withdrawal symptoms, control of cravings and heroin abstinence) on this dose.

Buprenorphine became available as a treatment option in 2005, initially as the sublingual mono buprenorphine tablets (Subutex ${ }^{\circledR}$, Reckitt Benckiser Healthcare [UK] Ltd). In 2007, we switched to use of the buprenorphine-naloxone combined preparation Suboxone ${ }^{\circledR}$ (Reckitt Benckiser Healthcare [UK] Ltd). Induction usually involved provision of 2mgs buprenorphine during the morning of day 1 , with a further 2 to $6 \mathrm{mgs}$ later that afternoon. Single daily doses of up to 8 to $12 \mathrm{mgs}$ were administered from day 2. Stabilisation doses were typically in the region of 6 to $12 \mathrm{mgs}$. Consistent with the recent guidelines on opiate substitution treatment, buprenorphine was offered where patients had shorter heroin use histories and were striving towards detox on the short to medium term (NICE, 2007; SAMSHA, 2009; Kaminer \& Marsch, 2011). We found that about $40 \%$ of patients were unwilling to agree to buprenorphine treatment when it was recommended by the clinical team. In such cases, we then offered methadone. 
Although published after this study, our approach to medication induction and stabilisation for these adolescent patients was consistent with that outlined in the recent British treatment guidelines on this topic (Department of Health, 2009).

If patients continued to use heroin or resumed use after a period of abstinence, an increase in dose of opiate substitution treatment was considered. They were also encouraged to participate in increased counselling sessions with a focus on functional analysis of drug use episodes and on relapse prevention skills (McKay, 2009). Where initial opiate substitution treatment option was associated with a poor treatment response, the patient was offered the option of switching to the alternative. If heroin use persisted despite these treatment changes, consideration was given to arranging transfer to the adult maintenance program.

Patients provided 2 supervised urine samples per week for drug toxicology during treatment. These samples were screened for opiates, EDDP (a methadone metabolite), cocaine, amphetamines, cannabis, benzodiazepines and alcohol. The provision of 'take-away' doses of medication were utilised as a contingent reinforcer of opiate abstinence, as evidenced by urine toxicology. As patients established a period of opiate abstinence, they incrementally earned increased 'take-away' doses of medication. After two weeks of heroin abstinence, they received one 'take-away' dose, typically on a Saturday or Sunday. Once they attained a period of four weeks abstinence, they obtained a second 'take-away' dose. A subsequent lapse to heroin use resulted in an immediate loss of one of their 'take-away' doses. Ongoing heroin use resulted in loss of all 'take-aways'. Patients were also provided with treatment of co-morbid physical or psychiatric problems. They were actively supported in addressing co-existing housing, vocational and criminal justice related needs as part of their care plan. If they obtained work or training or re-accessed education, they were provided with take-away doses of medication to facilitate their participation in same. Such patients typically attended the program just one or two days per week, receiving 'take-aways' for the remaining days. 
We wished to avoid disciplinary discharges, and only considered this option where clients were seriously jeopardising the safety of other patients via drug dealing on the premises and actual acts of violence towards others. Where clients presented with high levels of aggression, transfer to the adult treatment service was considered.

The service was harm reduction orientated. Nevertheless, it held an aspiration, but not an insistence, that all patients progress towards complete abstinence via detoxification. Prior to detoxification, patients typically spend a number of months stabilising on opiate substitution treatment, during which time they are expected to cease abuse of heroin and other drugs, while also addressing the co-existing psychological and social problems which might leave them vulnerable to relapse. Where detox, or dose tapering, occurred, it was generally conducted slowly on an outpatient basis over a period of about three months, negotiated with the individual patient. If the patient returned to heroin use during a detox, it was recommended that detox halt temporarily or else revert to a further period on a stabilisation dose. Where patients insisted on finishing a detox and leaving treatment despite evidence from urinalysis of ongoing heroin use, they were viewed as treatment drop outs. Patients had the option of referral to a residential specialist addiction treatment unit, under the care of the consultant child and adolescent psychiatrist, to commence and complete detox. In practice, about one third of the patients who complete detoxification utilise this latter option. Following detox, the YPP either provided ongoing psychological support or facilitated the patient in accessing same at an alternative, more locally accessible service. Where patients relapsed following detox, we strived to promptly re-engage them back into treatment.

\subsection{Patients.}

Although the YPP accepted referrals on young people who were opioid dependent, in this study we focused on heroin users specifically. We included all patients who were heroin 
dependent, who were treated with opiate substitution medication, and initiated treatment after the YPP was opened in May 2000. Patients with a primary diagnosis of dependence on prescription opioids were excluded. Patients who dropped out of the assessment process before commencing medication were not included. We included the first one hundred consecutive patients who met these criteria and we reached this number of recruits in July 2008. The end point for data collection on treatment outcome for each patient was the point at which they last exited treatment on the YPP or July 2009, whichever was earlier.

\subsection{Data Collection}

The study received ethical approval from the Ethics Committee of the Drug Treatment Centre Board. Baseline descriptive characteristics were obtained from the patients' initial structured assessment, this being adapted from the Maudsley Addiction Profile (Marsden et al, 1998). The assessment was administered by YPP staff. We recorded information on patient treatment participation three, six and twelve months after commencement on opiate substitution treatment. If a patient was not attending the service on these dates, we noted the date of and reason for exit. For patients in treatment on or after twelve months we recorded the final route of exit from the YPP, and the date of same.

The primary outcome of interest in this study was the final progression routes of patients. We categorised all patients into one of four different progression routes. Those who were referred to and commenced on another opiate substitution treatment program were categorised as "transfers for ongoing treatment". Patients were categorised as exiting via "detox" if they completed the prescribed detox regime and showed urinalysis evidence of opiate abstinence at discharge from the treatment program. Patients who ended treatment because they were incarcerated were categorised as exiting due to "prison". Finally, patients who simply stopped 
attending, relocated without arranging alternative treatment or left treatment prematurely against medical advice were all categorised as being "drop outs".

In order to examine mortality, we used the dates of treatment entry and final departure from the YPP to calculate person years at risk. For patients who exited the service within twelve months we sought to confirm that they were alive at twelve months by contacting them, local treatment services or the other professionals involved in their care.

For patients still in treatment at one year, we examined their success in avoiding heroin use by using the results of their twice weekly urinalysis during their twelfth month of treatment. For patients who had progressed out of treatment within twelve months, we did not seek to obtain information on their current drug use.

Viral serology was offered during the first few weeks of treatment. We screened for antibodies to HIV and hepatitis $\mathrm{C}$ using enzyme linked immunosorbent assays. Those with baseline negative results were offered repeat screens, about every nine to twelve months.

We reviewed records of all patients who presented for assessment to the YPP to identify those people who were heroin dependent, or probably heroin dependent, and began assessment for opiate substitution treatment but did not commence same. We gathered this information to determine the proportion of patients who dropped out of the assessment process.

As the YPP was not the only provider of opiate substitution treatment to adolescents in Dublin or the surrounding counties during this period, we obtained information from the Central Treatment List (CTL) on all people from this region under the age of 18 who were commenced on methadone during the study period. We obtained this information in order to better characterise treatment provision in Dublin and also to examine how representative the YPP 
study group was of the wider adolescent group in treatment in the Dublin region. In order to adhere to data protection guidelines, we received a breakdown of such patients by age and by gender. Information was provided on the number of distinct individuals who commenced treatment in each separate service. However, it was not possible to determine how many of these individuals received treatment also on the YPP or in other settings. For example, if an individual patient re-accessed treatment in the one service on three occasions, they featured only once in the CTL dataset provided. However, if they received treatment in three separate services, they featured three times in the dataset.

\subsection{Statistics}

We compared baseline characteristics and treatment progression route by gender. We used Pearson chi square test to explore association between categorical variable and gender, except in instances where a predicted cell value was less then 5, where Fisher's Exact test was utilised. For continuous variables we conducted the Independent samples t-Test.

When calculating the incidence of viral infection, the date of the first negative test represented the starting point for all patients when calculating their person years at risk (PYAR). The endpoint was the date of the last negative test for those who remained seronegative. The estimated date of seroconversion was used as the endpoint for those who seroconverted and this was calculated by finding the midpoint between their most recent negative test and their first positive. The $95 \%$ confidence interval (CI) was calculated by using the exact confidence intervals for binomial proportions.

\section{Results}

\subsection{Patient profile}


During the recruitment period, only 1 patient was excluded due to the fact that he was primarily dependent on methadone, which he purchased illicitly and he denied ever using heroin. No patient presented with primary dependence on other prescription opioid medications. There were a further 20 patients who presented with probable or definite heroin dependence, began assessment for treatment, but did not commence opiate substitution treatment on the YPP. Of these two were redirected to inpatient treatment and two were redirected to a more accessible local outpatient service. Four were incarcerated while attending for assessment and twelve either dropped out or opted not to pursue treatment. Although those who commenced assessment but did not start treatment on the YPP were no different in age from those who commenced treatment, they were more likely to be male (Odds Ratio 3.3, $\mathrm{p}=0.03)$

Of the 100 patients commenced on opiate substitution treatment on the YPP included in this study, 54 were female and 88 were aged 17 years or younger at treatment commencement. There were 13 patients aged under 16 years.

From the Central Treatment List (CTL), it emerged that there were 117 treatment episodes involving methadone treatment of a person aged 17 years and younger at other outpatient services in the region during this 8 year period. These treatment episodes are not reported in this study. As the information provided by the CTL relates to treatment episodes, we do not know how many of these episodes relate to people who were also treated in the YPP. Seventy of these treatment episodes were conducted at one of the four other dedicated youth treatment programs which existed in Dublin during this period. Nine episodes were initiated by GPs and the remaining 38 episodes occurred at adult treatment clinics. The mean age of the patients involved in these other 117 treatment episodes was 16.7 years (SD 0.7) and 48\% were female, these characteristics being very similar to the YPP cohort described in this study. 
The socio-demographic, clinical and treatment characteristics of the YPP patient group are reported in Table 1, in which gender differences are also highlighted. Males were more likely than females to present with a history of previous convictions, imprisonment, early school leaving and opiate abuse by a sibling. Females were more likely to be in a relationship with another heroin user and to have a history of deliberate self harm.

\section{Insert Table 1 here}

\subsection{Opiate substitution treatment}

Nineteen were prescribed buprenorphine and the others receiving methadone at treatment commencement. We examined the methadone dose for each patient in treatment after 3,6 and 12 months. Where patients were on methadone treatment at more than one of these time-points, we noted the highest or peak methadone dose for each individual. The mean 'peak' dose was 53mgs (range 15-90mgs). Of those initiated on buprenorphine, during the first three months on the YPP, 8 patients had a planned discharge following detox completion, 5 had dropped out, 4 had switched to methadone treatment. Only 2 people remained on this medication at 3 months (doses 4mgs and 12mgs). At 6 and 12 months, there was only one patient on buprenorphine at each occasion (doses $8 \mathrm{mgs}$ and $6 \mathrm{mgs}$ respectively).

\subsection{Treatment Outcome}

Table 2 highlights patients' progression out of and back into treatment. Of the 28 people who dropped out during the first year of treatment, 19 left during the first 3 months. Five of those very early dropouts subsequently returned to the YPP. While 21 people exited treatment following detox completion during the first 6 months of treatment, twelve of these subsequently returned back into treatment in the YPP following a relapse. 
At 3, 6 and 12 months after treatment commencement, 63, 55 and 50 patients were in treatment on the YPP respectively (see Table 2). Of the 50 patients who were in treatment after one year, urinalysis was available on 49 during their twelfth month of treatment. Of these, 19 demonstrated complete abstinence from heroin use during that month (see Table 1).

\section{Insert Table 2 here}

Ninety-two patients had progressed out of treatment from the YPP at the end of the study period. Of these, the overall proportion exiting via planned discharge following detox was $22 \%$. The proportions finally exiting via drop out and via imprisonment were $32 \%$ and $8 \%$ respectively. The proportion who were ultimately transferred to another setting for ongoing opiate substitution treatment was $39 \%$. There was just 1 disciplinary discharge. Treatment duration and the final progression route are reported in Table 1, by gender. Males were more likely than females to be imprisoned. Of the 36 transferred elsewhere for ongoing treatment, 7 had previously completed a detox but had relapsed.

The median time gap from first entry into treatment to final discharge from the YPP for those who exited following detox completion was 4 months (Range 10 days to 42 months [Interquartile Range $\{\mathrm{IQR}\} 7$ weeks to 19 months]). It was 8 months (Range 6 days to 30 months [IQR 3 to 13 months]) for those who dropped out of treatment and 23 months (Range 1 week to 44 months [IQR 15 to 31 months]) for those who transferred to another service for ongoing opiate substitution treatment.

\subsection{Incidence of HCV \& HIV infection}

Of the 84 people who agreed to undergo hepatitis C viral (HCV) serology, 17 (20\%) were antibody positive at baseline. Thirty-seven of those who initially tested negative were later retested and 6 seroconverted during 53.4 years of follow-up giving an incidence of 11 
infections/100 PYAR (95\% CI, 4 - 22/100PYAR). Of the 46 who reported a history of injecting, 36 were screened for HCV and $12(33 \%)$ were seropositive at baseline. The incidence of HCV among the 19 injectors re-tested was three infections in 21.5 person years or 14/100PYAR (95\% CI, 3-35/100 PYAR). All of the 69 patients tested for HIV at baseline were negative. Of these, 31 underwent at least one further test on the YPP during 42.3 years of follow-up and all remained seronegative.

\subsection{Mortality}

There were no deaths during treatment among these 100 patients who had a cumulative period of 129 person years at risk. Fifty patients left treatment within 12 months of starting on the YPP. Of these we were able to confirm that 47 were alive at 12 months, but there was no information on the status of the other 3 patients.

\section{Discussion}

\subsection{Patient Characteristics}

The patient population showed evidence of quite profound psycho-social adversity at baseline in common with similar patient groups across the world (Crome et al, 2000; Millman, Khuri \& Nyswander, 1978). The slight excess of females in this age range contrasts with adult populations of heroin users where males account for about $70 \%$ of treatment contacts in Ireland (Carew, 2009). In Australia, (Bell \& Mutch 2006) also found that females outnumbered males in their cohort. In USA, the gender profile among adolescent heroin users accessing treatment was almost equal (SAMSHA, 2009).

\subsection{Opiate Detoxification}

Most teenage heroin users themselves, and nearly all referrers and parents, aspire to a goal of abstinence (Crome et al, 2000). Despite holding an ambition that the patients attending the YPP would ultimately detox, only $22 \%$ left the treatment service via this route. Many others 
who completed a detox relapsed quite quickly and re-accessed opiate substitution treatment again. While abstinence proved somewhat elusive for our young patients, it should be borne in mind that a pattern of relapse and multiple treatment episodes is commonplace across all severe addictions in this age range (Fishman, 2011). Adult heroin users also struggle to attain this goal. In a recent study of treatment outcome among over 18,000 adult heroin users in England, Marsden et al (2009) found that just 5\% had a planned discharge from medication assisted treatment within their first year of treatment. In Ireland it is estimated that just $1 \%$ of adults on methadone treatment exit via a planned detox each year (Comptroller \& Auditor General, 2009).

The proportion progressing from treatment via planned detox is less than the $29 \%$ reported by Millman, Khuri \& Nyswander (1978). Those who detoxed had spent a mean period of 28 months on methadone in their study in New York. Their service therefore worked with patients for even longer periods than occurred on the YPP. They appear to have had a much less dispersed catchment area than that which exists on the YPP, perhaps permitting them to provide more active and ongoing support to those who completed detox.

\subsection{Treatment Drop-out and imprisonment}

In common with others, we found that drop-out from treatment was commonplace, especially during the first three months of treatment (Millman, Khuri \& Nyswander 1978; Marsch et al 2005; Bell \& Mutch 2006; Kellogg et al, 2006). A third of the early drop-outs did subsequently re-enter treatment in our population. Due to the more generous staffing of the YPP, it can provide higher levels of program structure, patient support and monitoring than the typical community based adult addiction treatment services in Dublin. While most patients welcome this and it may assist patient retention and re-engagement, it is possible that others prefer the less demanding treatment approaches used in other adult orientated services and many YPP 
drop-outs may have subsequently re-accessed treatment in those settings, especially following their $18^{\text {th }}$ birthday.

Despite the frequent history of extensive criminality at baseline, less than $10 \%$ were imprisoned while in treatment. We view this as a qualified success for the service. Males were more likely to have a history of incarceration prior to treatment entry and were more likely to exit treatment via imprisonment. There were no other gender differences in outcomes.

\subsection{Ongoing Opiate Substitution Treatment}

We found that the most common progression route was transfer to the adult addiction service for ongoing treatment. While this ensures that patients continue to benefit from the harm reducing benefits of opiate substitution treatment, it does come as a disappointment to many teenagers and to their relatives. Again it reflects international experience and the reality that many young patients require a longer period of treatment than that offered on the YPP (Millman, Khuri \& Nyswander, 1978; Bell \& Mutch 2006). Many patients arrived into the YPP with evidence of major coexisting difficulties including poor education attainment, family histories of opiate and alcohol abuse and significant comorbid mental health difficulties. In light of these problems, the challenge of attaining and sustaining complete opiate abstinence is very substantial. Woody et al (2008) reported that the majority of opioid dependent youth quickly returned to drug use following detox completion and argued that prolonged opiate substitution treatment will be necessary for many young patients. Continuity of treatment is a key predictor of patient outcome and our successful efforts to actively facilitate transfer into ongoing treatment are therefore important (NICE, 2007; Fishman, 2011).

\subsection{Impact on Heroin Use}

Urinalysis indicated that over one third of our patients on treatment at twelve months were completely abstinent from heroin and there was evidence of reductions in heroin use in a 
further half of patients. This suggests that this treatment may indeed be effective in reducing heroin use in this young age range. While there have been a small number of recent rigorous examinations of heroin use after one to three-months on opiate substitution treatment in youth, there has been a paucity of literature looking at longer term outcome (Minozzi, Amato \& Davoli, 2009). We share the view that many heroin dependent adolescents require treatments of at least one year duration and more long term outcome studies are needed (Hopfer et al, 2002).

\subsection{Blood Borne Viral Infection Outcomes}

It is well established that MMT results in reductions in unsafe injecting in adults (Marsch, 1998). We found that the incidence of HCV was just 14/100PYAR among our very young subgroup of injecting drug users (IDU). Younger age is a recognised risk factor for increased HCV incidence among IDU (van Beek et al, 1998). While our sample size is very small, our finding compares favourably with an earlier Dublin study which reported a HCV incidence among IDU of 66 infections/100PYAR in the 1990s (Smyth et al, 2003).

\subsection{No Deaths Observed}

While there have been some small scale studies of opiate substitution treatment in adolescents, its safety is not well established (Minozzi, Amato \& Davoli, 2009; O’Brien, 2005). There were no deaths among those in treatment at the YPP during this study. It is during the months following treatment exit, whether by planned detoxification or drop out, that mortality is seen to rise in adult populations (Fugelstad et al, 2006). Among those who left treatment within twelve months of starting on the YPP, we were able to confirm that they were alive on their one year anniversary of treatment commencement in $94 \%$ of cases. Our study was not powered to draw any firm conclusions on the safety of this treatment in adolescents or its impact on mortality, but we did not detect any evidence that it is particularly hazardous. 


\subsection{Limitations}

The limitations of the study include its naturalistic design and limited information on those patients who were not in treatment at each of the time points examined. Use of a validated assessment instrument such as the MAP would have assisted in permitting easier international comparison (Marsden et al, 1998). Although our findings indicate the dynamic nature of treatment departure and re-entry, the method of data collection will have under-estimated this phenomenon. For example, if a patient dropped out of treatment or detoxed during month eight and subsequently returned in month ten, then this will not have been captured as we ascertained location on month six and month twelve at which points such an patient would have been in treatment. While we have provided urinalysis data for those who were still in treatment at twelve months, we did not obtain any outcome information of opiate use among those who departed treatment in a planned or unplanned manner prior to this point. Our finding that $22 \%$ of patients exited treatment following detox completion cannot be interpreted as indicating that this proportion attained long term abstinence as outcome after discharge was not measured. National and international experience indicates that many will have relapsed within months of discharge from the YPP (NICE, 2007).

The study only examined outcome of those who persisted with the relatively long assessment process and succeeded in commencing medication treatment. The clinical decision to require three assessment appointments was made on the basis of a desire to avoid a situation whereby non-dependent patients might inappropriately commence treatment. The vast majority of patients negotiated this hurdle, with just 12 heroin dependent patients dropping out of the assessment process. Nevertheless, a faster journey into treatment may have resulted in more study participants and may have altered the detected outcome profile.

The treatment provided to patients was heterogeneous and tailored to their individualised care plan. While this is the nature of most clinical practice, it does nevertheless complicate 
interpretation of our outcome findings. We have provided substantial information on the approach to treatment to assist the reader in determining how applicable our findings may be to other treatment settings. The methadone doses, averaging 50mgs, may be seen to be insufficient. However, this relatively low average dose reflects the fact that many patients were undergoing detoxification at each time point and doses of up to 90mgs were prescribed where indicated.

While information from the CTL indicates that our patient group is representative of treated opiate adolescents in Dublin, it is a relatively small study sample and contrasts starkly with the large sample sizes included in some studies of treatment outcome in adult populations of heroin users (Marsden et al, 2009). While this is the nature of research studies on adolescents, it does nevertheless hamper interpretation of our findings, especially those relating to events which are quite rare, such as deaths, HIV and Hepatitis C seroconversion. While clinical trials represent the gold standard for outcome research there are enormous practical difficulties conducting such trials within this adolescent patient group to examine interventions of appropriately long term duration (Minozzi, Amato \& Davoli, 2009; Woody et al, 2008). Even in a country as vast as USA, only 50 adolescent heroin users were due to commence methadone or buprenorphine in 2007 (SAMSHA, 2009).

\subsection{Implications for service delivery}

Heroin dependence represents a complex and serious clinical problem and poses major treatment challenges. While many adolescent patients enter opiate substitution treatment with an aspiration to detox and leave treatment, our findings, and those of others, indicate that this goal is rarely attained. Services must be prepared for a significant challenge in engaging and retaining this patient group in treatment as about one third will drop out at least once, especially early in treatment. In common with adult patient groups, most of those who remain in treatment demonstrate reductions in heroin use. In view of the relatively large proportion of 
patients who never complete a detox and the relapses that occur among those who do, patients, parents and referrers should be aware that opiate substitution treatment will be ongoing beyond one year for close to half of all of these very young patients. 


\section{References}

Bell, J. \& Mutch, C. (2006). Treatment retention in adolescent patients treated with methadone or buprenorphine for opioid dependence: a file review. Drug and Alcohol Review, 25, 167-171.

Bulter, S. (2002). The making of the methadone protocol: the Irish system? Drugs: education, prevention and policy, 9, 311-324.

Carew, A.M., Bellerose, D., Lyons, S \& Long, J. (2009). Trends in treated problem opiate use in Ireland, 2002 to 2007. Dublin: Health Research Board.

Center for Substance Abuse Treatment. (2005). Initial screening, admission procedures, and assessment techniques. In: Batki SL, Kauffman JF, Marion I, Parrino MW, Woody GE, Medication-assisted treatment for opioid addiction in opioid treatment programs. (pp 43-61) Treatment improvement protocol (TIP) Series. DHHS Publication No. (SMA) 05-4048. Rockville (MD): Substance Abuse and Mental Health Services Administration.

College of Physicians \& Surgeons of Ontario. (2005). Methadone Maintenance Guidelines. Toronto, Ontario: College of Physicians \& Surgeons of Ontario

Comptroller and Auditor General (2009). Drug Addiction Treatment and Rehabilitation. Dublin: Department of Community, Rural and Gaeltacht Affairs. http://www.audgen.gov.ie/documents/vfmreports/64_Drug_Addiction_and_Rehab.pdf (accessed 7 December 2010) 
Crome, I.B., Christian, J. \& Green, C. (2000). The development of a unique designated community drug service for adolescents: policy prevention education implication. Drugs: Education, Prevention and Policy, 7, 87-108.

Department of Health (2009). Guidance for the pharmacological management of substance misuse among young people. London: Department of Health. http://www.dh.gov.uk/prod_consum_dh/groups/dh_digitalassets/documents/digitalasset/dh_10 6429.pdf (accessed October 12, 2009)

Dept of Health \& Children (2005) Report of the Working Group on Treatment of under $18 \mathrm{~s}$ presenting to Treatment services with Serious Drug Problems. Dublin: Dept of Health \& Children, Dublin.

Fishman M (2011) Placement criteria and treatment planning for adolescents with substance use disorders. In Eds. Kaminer Y, Winters KC. Clinical Manual of Adolescent Substance Abuse Treatment. Washington, DC: American Psychiatric Publishing

Fudala, P.J. \& Woody, G.W. (2004). Recent advances in the treatment of opiate addiction. Current Psychiatry Reports, 6, 339-346.

Fugelstad, A., Stenbacka, M., Leifman, A., Nylander, M. \& Thiblin, I. (2006). Methadone maintenance treatment: The balance between life-saving treatment and fatal poisonings. Addiction, 102, 406-412.

Gonzales, R., Brecht, M. Mooney, L., Rawson, R.A. (2011) Prescription and over-the-counter drug treatment admissions to the California public treatment system. Journal of Substance Abuse Treatment, 40, 224-229. 
Hopfer, C.J., Khuri, E., Crowley, T.J. \& Hooks, S. (2002). Adolescent heroin use: a review of the descriptive and treatment literature. Journal of Substance Abuse Treatment, 23, 231-237.

Kaminer Y, Marsch LA (2011) Pharmacotherapy of adolescent substance use disorders. In Eds. Kaminer Y, Winters KC. Clinical Manual of Adolescent Substance Abuse Treatment. Washington, DC: American Psychiatric Publishing

Kellogg, S., Melia, D., Khuri, E., Lin, A., Ho, A. \& Kreek, M.J. (2006) Adolescent and young adult heroin patients: Drug use and success in methadone maintenance treatment. Journal of Addictive Disease, 25, 15-25.

Lehmann, W.X (1973). The use of 1-alpha-acetyl-methadol (LAAM) as compared to methadone in the maintenance and detoxification of young heroin addicts. NIDA monograph, $8,82-3$.

Marsch, L. (1998). The efficacy of methadone maintenance interventions in reducing illicit opiate use, HIV risk behavior and criminality: a meta-analysis. Addiction, 93, 515-532.

Marsch, L.A., Bickel, W.K., Badger, G.J., Stothart, M.E., Quesnel, K.J., Stanger, C., Brooklyn, J. (2005). Comparison of pharmacological treatments for opioid-dependent adolescents: a randomized controlled trial. Archives of General Psychiatry, 62, 1157-1164.

Marsden, J., Gossop, M., Stewart, D. Best, D., Farrell, M., Lehmann, P., Edwards, C., Strang, J. (1998) The Maudsley Addiction Profile (MAP): a brief instrument for assessing treatment outcome. Addiction, 93, 1857-1868. 
Marsden, J., Eastwood, B., Bradbury, C, Dale-Perera, A., Farrell, M., Hammond, P., Knight, J., Randhawa, K., Wright, C. (2009). Effectiveness of community treatments for heroin and crack cocaine addiction in England: a prospective, in-treatment cohort study. Lancet, 374, 12621270.

McKay, J.R. (2009). Continuing care research: what we have learned and where we are going. Journal of Substance Abuse Treatment, 36, 131-145.

Millman, R.B., Khuri, E.T. \& Nyswander, M.E. (1978). Therapeutic detoxification of adolescent heroin addicts. Annals of the New York Academy of Science, 311, 153-164

Minozzi, S., Amato, L. \& Davoli, M. (2009). Maintenance treatments for opiate dependent adolescent. Cochrane Database of Systematic Reviews, Issue 2. Art. No.: CD007210. DOI: 10.1002/14651858.CD007210.pub2.

Motamed, M, Marsch, LA,, Solhkhah, R, Bickel, WK, Badger, GJ. (2008) Differences in Treatment Outcomes between Prescription Opioid-Dependent and Heroin-Dependent Adolescents. Journal of Addiction Medicine, 2, 158-164

NICE (2007) Methadone and buprenorphine for the management of opioid dependence. London: National Institute for Health \& Clinical Excellence.

NTA (2009) Getting to grips with substance misuse among young people. London: National Treatment Agency for Substance Misuse.

http://www.nta.nhs.uk/uploads/nta_young_peoples_report_2009.pdf (accessed 24 June 2010)

O’Brien, C.P. (2005). Adolescent Opioid Abuse. Archives of General Psychiatry, 62, 1165. 
SAMSHA (2009) The TEDS Report: Characteristics of adolescent heroin admissions.

Rockville, MD: Substance Abuse \& Mental Health Service.

Sells, S.B. \& Simpson, D.D. (1979). Evaluation of treatment outcome for youths in the Drug Abuse Reporting Program (DARP): a follow-up study. In G.M. Beschner \& A.A. Friedman (Eds.), Youth drug abuse: problems, issues and treatment (pp571-622). Lanham, MD: Lexington Books.

Smyth, B.P., O’Connor, J.J., Barry, J. \& Keenan, E. (2003). Retrospective cohort study examining incidence of HIV and hepatitis $\mathrm{C}$ infection among injecting drug users in Dublin. Journal of Epidemiology \& Community Health, 57, 310-311.

Woody, G.E., Poole, S.A., Subramaniam, G., Dugosh, K., Bogenschutz, M., Abbott, P., Patkar, A., Publicker, M., McCain, K., Potter, J.S., Forman, R., Vetter, V., McNicholas, L., Blaine, J., Lynch, K.G., Fudala, P (2008). Extended vs short-term buprenorphine-naloxone for treatment of opioid-addicted youth: a randomized trial. Journal of the American Medical Association, 300, 2003-2011.

van Beek, I., Dwyer, R., Dore, G.J., Luo, K., Kaldor, J.M. (1998). Infection with HIV and hepatitis $\mathrm{C}$ virus among injecting drug users in a prevention setting: retrospective cohort study. British Medical Journal, 317, 433-437 
Table 1. Characteristics of 100 adolescents presenting for opiate substitution treatment, by gender

\begin{tabular}{|c|c|c|c|c|c|c|c|}
\hline \multirow[t]{2}{*}{ Characteristic } & \multicolumn{2}{|c|}{ Total Group } & \multicolumn{2}{|c|}{ Males $(n=46)$} & \multicolumn{2}{|c|}{ Females $(n=54)$} & \multirow[b]{2}{*}{ p value ${ }^{a}$} \\
\hline & $\mathrm{N}(\%)$ & $\begin{array}{l}\text { Mean } \\
(\mathrm{SD})\end{array}$ & $\mathrm{N}(\%)$ & $\begin{array}{l}\text { Mean } \\
\text { (SD) }\end{array}$ & $\mathrm{N}(\%)$ & $\begin{array}{l}\text { Mean } \\
\text { (SD) }\end{array}$ & \\
\hline \multicolumn{8}{|l|}{ Demographics, Supports \& Criminality } \\
\hline Age in Years & & $16.6(0.9)$ & & $16.6(0.8)$ & & $16.6(1.0)$ & NS \\
\hline Age last attended education & & $14.4(1.6)$ & & $13.8(1.8)$ & & $14.9(1.2)$ & 0.002 \\
\hline In School or training & $11(11)$ & & $6(11)$ & & $5(11)$ & & NS \\
\hline Two parent family support & $53(54)$ & & $21(46)$ & & $32(60)$ & & NS \\
\hline Parental alcohol abuse & $55(60)$ & & $25(60)$ & & $30(60)$ & & NS \\
\hline Sibling Opiate abuse & $41(43)$ & & $25(56)$ & & $17(32)$ & & 0.02 \\
\hline Ever in care & $29(30)$ & & $17(38)$ & & $12(23)$ & & NS \\
\hline Homeless in past month & $30(30)$ & & $14(30)$ & & $16(30)$ & & NS \\
\hline Previous criminal convictions & $40(43)$ & & $26(59)$ & & $14(29)$ & & 0.004 \\
\hline Ever incarcerated & $25(27)$ & & $18(41)$ & & $7(14)$ & & 0.004 \\
\hline Current boyfriend/girlfriend using heroin & $41(42)$ & & $7(16)$ & & $34(64)$ & & $<0.001$ \\
\hline \multicolumn{8}{|l|}{ Psychiatric History } \\
\hline Ever seen a psychiatrist & $49(50)$ & & $25(56)$ & & $24(45)$ & & NS \\
\hline History of deliberate self harm & $29(30)$ & & $8(18)$ & & $21(41)$ & & 0.01 \\
\hline \multicolumn{8}{|l|}{ Substance Use } \\
\hline Age of first heroin use & & $14.7(1.3)$ & & $14.6(1.6)$ & & $14.7(1.1)$ & NS \\
\hline Months of regular heroin use & & $16.5(11.5)$ & & $18.3(12.3)$ & & $14.9(10.8)$ & NS \\
\hline Number of 'bags' of heroin per day & & $3.5(1.8)$ & & $3.6(1.9)$ & & $3.5(1.8)$ & NS \\
\hline Past Month Drug Use & & & & & & & \\
\hline Benzodiazepine misuse & $54(55)$ & & $26(57)$ & & $28(54)$ & & NS \\
\hline Use of black market methadone & $50(53)$ & & $25(57)$ & & $25(49)$ & & NS \\
\hline Cocaine use & $18(19)$ & & $9(20)$ & & $9(18)$ & & NS \\
\hline Ever injected & $46(47)$ & & $23(51)$ & & $23(43)$ & & NS \\
\hline \multicolumn{8}{|l|}{ Hepatitis C testing } \\
\hline Untested for $\mathrm{HCV}$ & $16(16)$ & & $8(17)$ & & $8(15)$ & & NS \\
\hline $\mathrm{HCV}$ antibody positive at baseline ${ }^{\mathrm{b}}$ & $17(20)$ & & $8(21)$ & & $9(20)$ & & NS \\
\hline Initial treatment with buprenorphine & $19(19)$ & & $9(20)$ & & $10(19)$ & & NS \\
\hline \multicolumn{8}{|l|}{ Treatment duration } \\
\hline Months in contact with treatment service ${ }^{c}$ & & $14.5(12.1)$ & & $13.4(12.0)$ & & $15.4(12.3)$ & NS \\
\hline Still in treatment at 12 months & $50(50)$ & & $19(41)$ & & $31(57)$ & & NS \\
\hline \multicolumn{8}{|l|}{ Heroin Use during $12^{\text {th }}$ month of treatment $\mathrm{d}^{\mathrm{d}}$} \\
\hline Constant use & $7(14)$ & & $4(22)$ & & $3(10)$ & & NS \\
\hline Intermittent use & $23(47)$ & & $9(50)$ & & $14(45)$ & & NS \\
\hline Abstinent & $19(39)$ & & $5(28)$ & & $14(45)$ & & NS \\
\hline \multicolumn{8}{|l|}{$\begin{array}{l}\text { Route of discharge among } 92 \text { patients who } \\
\text { finished treatment }\end{array}$} \\
\hline Dropped out & $29(32)$ & & $14(33)$ & & $15(31)$ & & NS \\
\hline Transferred for ongoing treatment & $36(39)$ & & $16(37)$ & & $20(41)$ & & NS \\
\hline Planned discharge after detox completion & $20(22)$ & & $7(16)$ & & $13(27)$ & & NS \\
\hline Prison & $7(8)$ & & $6(14)$ & & $1(2)$ & & $0.05^{\mathrm{e}}$ \\
\hline
\end{tabular}

\footnotetext{
a The reported test statistic is the chi square value for categorical variable and Students $\mathrm{t}$ test statistic for quantitative variables. "NS" = Not significant

${ }^{\mathrm{b}}$ Proportion HCV antibody positive in initial HCV screen, among those tested for same.

${ }^{c}$ This represents the number of months from first commencing treatment to final departure from the service.

d Heroin use determined via urinalysis. "Constant use" implies all urine drug screens were opiate positive. "Intermittent use" implies some, but not all, urine drug screens were opiate positive and "Abstinent" implies all urine drug screens were opiate negative.

${ }^{\mathrm{e}} \mathrm{P}$ value calculated using Fishers Exact Test Statistic as estimated value in cell was less than 5
} 


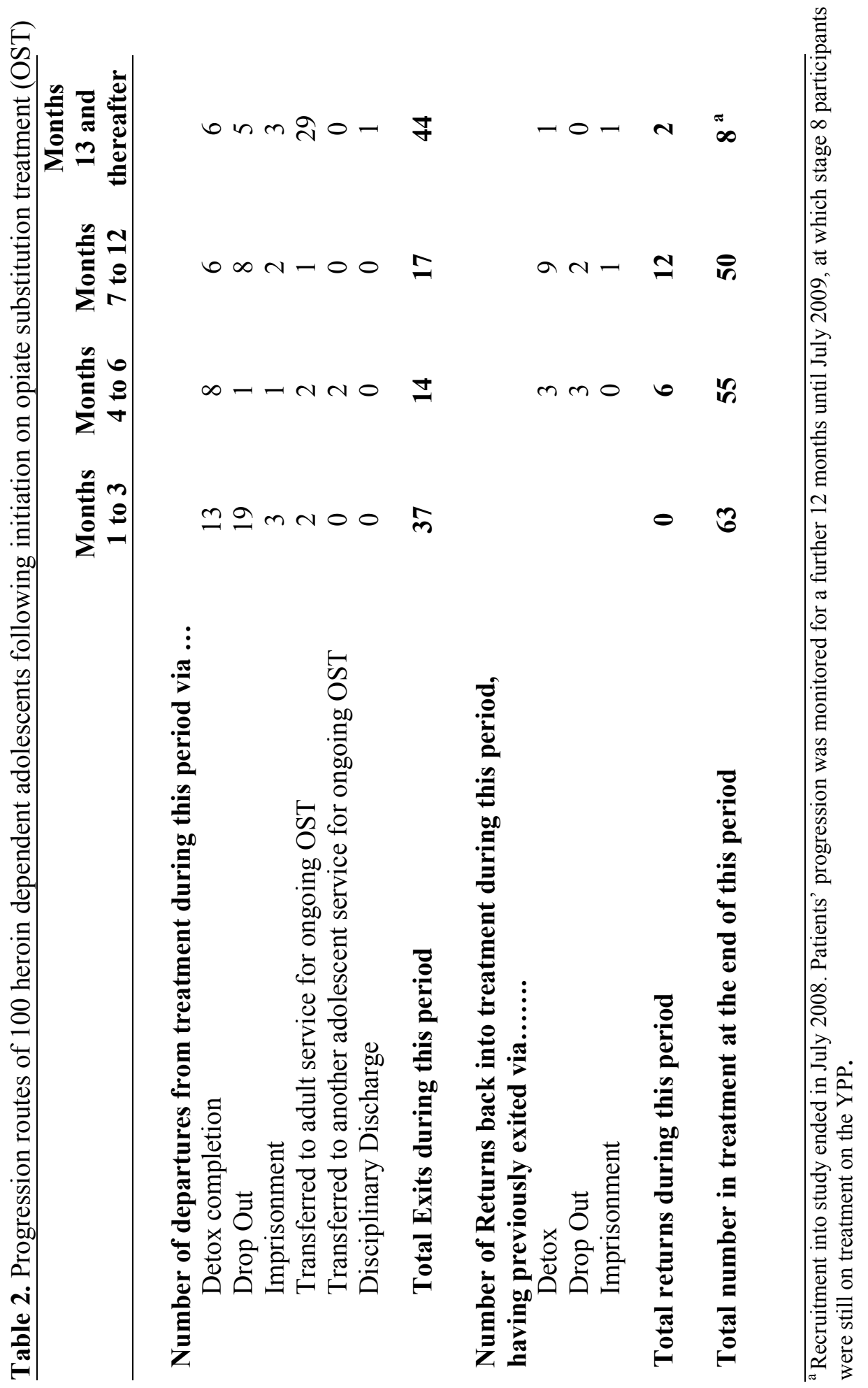

